\title{
Qualitative Analysis for a Reaction-Diffusion Predator-Prey Model with Disease in the Prey Species
}

\author{
Meihong Qiao, ${ }^{1}$ Anping Liu, ${ }^{1}$ and Urszula Forys ${ }^{2}$ \\ ${ }^{1}$ School of Mathematics \& Physics, China University of Geoscience, Wuhan 430074, Hubei Province, China \\ ${ }^{2}$ Institute of Applied Mathematics and Mechanics, Faculty of Mathematics, Informatics, \& Mechanics, University of Warsaw, \\ Banacha 2, 02-097 Warsaw, Poland
}

Correspondence should be addressed to Meihong Qiao; qiaomeihong@126.com

Received 3 February 2014; Accepted 8 April 2014; Published 29 April 2014

Academic Editor: Zhijun Liu

Copyright (c) 2014 Meihong Qiao et al. This is an open access article distributed under the Creative Commons Attribution License, which permits unrestricted use, distribution, and reproduction in any medium, provided the original work is properly cited.

\begin{abstract}
A diffusive predator-prey system with disease in predator species and no-flux boundary condition is considered. Sufficient conditions which ensure persistence of the system are obtained. Conditions of disease-free ecosystem are also studied. Furthermore, sufficient conditions for global asymptotic stability of the unique positive equilibrium and disease-free equilibrium of the system are derived using the approach of Lyapunov function.
\end{abstract}

\section{Introduction}

Ecoepidemiology is a relatively new branch of study in theoretical biology, which tackles problems by dealing with both ecological and epidemiological approach. It can be viewed as the coupling of an ecological predator-prey or competition model with an epidemiological SI, SIS, or more complex model. Anderson and May [1] were the first who marked that the effect of disease in ecological systems is an important issue from both mathematical and ecological point of view. They proposed an ecoepidemiological model by merging the ecological predator-prey model introduced by Lotka and Volterra with epidemiological model.

Clearly, in the natural world, species does not exist alone. While the disease is spread within the species, the species also competes with other species for environmental resources like space or food, or is predated by other species. Therefore, it is of more biological significance to consider the effect of interacting species when we study the dynamical behaviors of epidemiological models.

Many papers have been devoted to study the effects of a disease on a predator-prey system. Venturino [2] studied SI and SIS models with disease spread among the prey when the logistic growth of both the prey and predator populations is assumed and the predators eat infected preys only. In [3], Hsu and Huang considered the following predator-prey model:

$$
\begin{aligned}
& \frac{d u}{d t}=r u\left(1-\frac{u}{K}\right)-p(u) v, \\
& \frac{d v}{d t}=s v\left(1-\frac{h v}{u}\right),
\end{aligned}
$$

where $u$ and $v$ represent densities of the populations of prey and predators, respectively, and $r, s, K$, and $h$ are positive constants. The population of prey grows logistically with carrying capacity $K$ and intrinsic growth rate $r$ in the absence of predation. Predators consume prey according to the functional response $p(u)$ and grow logistically with intrinsic growth rate $s$. Carrying capacity of the predator species is proportional to the size of the prey population. It should be noticed that the model described by (1) is a generalisation of the prey-predator model proposed by May [4] which is known as Holling-Tanner model. In this model the functional response $p(u)=a u /(b+u)$ is of the Holling type [5], and it is one of the prototype models involving limit cycle dynamics. 


\section{Model Formulation}

On the basis of (1) we propose an ecoepidemiological model with a disease spread in the predator population. We assume that only predator can be infected and the infected individual does not recover or become immune. Because the predation ability of healthy (and susceptible at the same time) predators is stronger than infected ones, we suppose that prey can be preyed on only by healthy predators. Moreover, we also assume the simplest linear form of functional response $p(u)=k u$. Therefore, the model reads

$$
\begin{aligned}
& \frac{d u}{d t}=r u\left(1-\frac{u}{K}\right)-k u v \\
& \frac{d v}{d t}=s v\left(1-\frac{h v}{u}\right)-\beta w v \\
& \frac{d w}{d t}=\beta w v-d w-\mu w^{2}
\end{aligned}
$$

where $u, v$, and $w$ represent densities of the populations of prey, susceptible predator, and infected predator, respectively. The death rate of infected predators equals $d, \beta$ is the infectious rate of the disease, and $\mu$ is the density-dependent death rate of infected predators. Other parameters are the same as in (1).

Species dispersal is one of the most prevalent phenomena of nature, and many empirical studies and monographs on population dynamics in a spatial heterogeneous environment have been done (see [6-15] and the references cited therein). Most important subjects of population diffusion models are coexistence of populations, local and global stability of equilibria, existence of periodic solutions, and so forth, (see [1620]). In particular, single population models were considered, for example, in [21-23], while predator-prey system with the prey dispersal was studied, for example, in [24-26]. Such type of model is still of great interest and importance; compare the recent papers [27-30] and the references therein.

Taking into account inhomogeneous distribution of predators and their prey in different spatial locations within a fixed bounded domain $\Omega$ in $\mathbb{R}^{N}$ with smooth boundary at any given time and the natural tendency of each species to diffuse to areas of smaller population density, we are led to consider the following reaction-diffusion system:

$$
\begin{aligned}
\frac{\partial u}{\partial t} & =d_{1} \Delta u+r u\left(1-\frac{u}{K}\right)-k u v, \quad x \in \Omega, \\
\frac{\partial v}{\partial t} & =d_{2} \Delta v+s v\left(1-\frac{h v}{u}\right)-\beta w v, \quad x \in \Omega, \\
\frac{\partial w}{\partial t} & =d_{3} \Delta w+\beta w v-d w-\mu w^{2}, \quad x \in \Omega, \\
\frac{\partial u}{\partial \eta} & =\frac{\partial v}{\partial \eta}=\frac{\partial w}{\partial \eta}=0, \quad x \in \partial \Omega, \\
u(x, 0) & =u_{0}(x) \geq 0, \quad v(x, 0)=v_{0}(x) \geq 0, \\
w(x, 0) & =w_{0}(x) \geq 0, \quad x \in \Omega,
\end{aligned}
$$

where $\Omega$ is a bounded domain in $\mathbb{R}^{N}(N=2$ or 3 in reality) with smooth boundary $\partial \Omega, \partial / \partial \eta$ is the outward derivative normal to $\partial \Omega$, and $d_{1}, d_{2}$, and $d_{3}$ are strictly positive diffusion coefficients.

To reduce the number of parameters we make the following change of variables:

$$
r t \longmapsto t, \quad \frac{u}{K} \longmapsto u, \quad b v \longmapsto v, \quad \mu w \longmapsto w,
$$

and we rename parameters accordingly,

$$
\begin{gathered}
\frac{k}{r} \longmapsto k, \quad \frac{s}{r} \longmapsto s, \quad \frac{s h}{r K} \longmapsto b, \quad \frac{d_{1}}{r} \longmapsto d_{1}, \\
\frac{d_{2}}{r} \longmapsto d_{2}, \quad \frac{d_{3}}{r} \longmapsto d_{3}, \quad \frac{\beta}{r} \longmapsto \beta, \\
\frac{d}{r} \longmapsto d, \quad \frac{\mu}{r} \longmapsto \mu,
\end{gathered}
$$

obtaining nondimensional version of the model

$$
\begin{aligned}
\frac{\partial u}{\partial t} & =d_{1} \Delta u+u(1-u)-k u v, \quad x \in \Omega, \\
\frac{\partial v}{\partial t} & =d_{2} \Delta v+v\left(s-\frac{v}{u}\right)-\beta w v, \quad x \in \Omega, \\
\frac{\partial w}{\partial t} & =d_{3} \Delta w+\beta w v-d w-w^{2}, \quad x \in \Omega, \\
\frac{\partial u}{\partial \eta} & =\frac{\partial v}{\partial \eta}=\frac{\partial w}{\partial \eta}=0, \quad x \in \partial \Omega, \\
u(x, 0) & =u_{0}(x) \geq 0, \quad v(x, 0)=v_{0}(x) \geq 0, \\
w(x, 0) & =w_{0}(x) \geq 0, \quad x \in \Omega .
\end{aligned}
$$

In this paper we assume that $N=1$ as calculations are simpler in such a case. However, the results presented below can be extended for $N>1$. Our goal is to give conditions guaranteeing persistence of the ecosystem described by (6). The persistence means that the disease is spread and endemic equilibrium appears. Equations (6) have positive (endemic) equilibrium $\left(u^{*}, v^{*}, w^{*}\right)$, where

$$
\begin{aligned}
& u^{*}=\frac{1}{2 \beta^{2}}\left(\beta^{2}-k d \beta-k s-1\right. \\
& \left.\quad+\sqrt{\left(\beta^{2}-k d \beta-k s-1\right)^{2}+4 \beta^{2}}\right), \\
& v^{*}=\frac{1-u^{*}}{k}, \quad w^{*}=\beta v^{*}-d .
\end{aligned}
$$

Notice that $v^{*}>0 \Leftrightarrow u^{*}<1$ and $w^{*}>0 \Leftrightarrow v^{*}>d / \beta \Leftrightarrow$ $u^{*}<1-d k / \beta$, which means that $\beta>d k$ is the necessary condition for the existence of the positive equilibrium.

One can easily check that $u^{*}<1$, while the inequality $v^{*}>d / \beta$ is equivalent to $\beta>d k+d / s$.

On the other hand, it is also important to know conditions for disease-free ecosystem. Assuming that $\bar{w}=0$ we obtain semitrivial disease-free equilibrium $(\bar{u}, \bar{v}, 0)$ with

$$
\bar{u}=\frac{1}{1+k s}, \quad \bar{v}=s \bar{u} .
$$


Remark 1. For any parameter values (6) have the semitrivial equilibrium DFE with coordinates $(\bar{u}, \bar{v}, 0)$.

If $\beta>d k+(d / s)$, then (6) have the unique positive equilibrium $\mathrm{EE}$ with coordinates $\left(u^{*}, v^{*}, w^{*}\right)$.

In the next sections we focus on the analysis of (6) and propose conditions for global stability of EE. We start from the case without diffusion $\left(d_{i}=0\right.$ for $\left.i=1,2,3\right)$ and then turn to the system with positive diffusion coefficients.

\section{The Model without Diffusion}

In this section we assume $d_{i}=0, i=1,2,3$; that is, consider spatially homogenous case

$$
\begin{aligned}
\dot{u} & =u(1-u)-k u v, \\
\dot{v} & =v\left(s-\frac{v}{u}\right)-\beta w v, \\
\dot{w} & =\beta w v-d w-w^{2} .
\end{aligned}
$$

It is obvious that local solutions of (9) exist and are unique for any positive initial data $\left(u_{0}, v_{0}, w_{0}\right)$. Moreover, if $w_{0}=0$, then $w(t)=0$ for all $t \geq 0$. Hence, we assume that some infected predators appear at $t=0$ and we want to know if the disease spreads in the ecosystem.

Basing on the positivity of solutions we obtain the following estimates:

$$
\dot{u} \leq u(1-u) \Longrightarrow u(t) \leq u_{M}:=\max \left\{u_{0}, 1\right\},
$$

which yields

$$
\dot{v} \leq v\left(s-\frac{v}{u_{M}}\right) \Longrightarrow v(t) \leq v_{M}:=\max \left\{s u_{M}, v_{0}\right\},
$$

and therefore

$$
\begin{aligned}
\dot{w} & \leq w\left(\beta v_{M}-d-w\right) \Longrightarrow w(t) \\
& \leq w_{M}:=\max \left\{\beta v_{M}-d, w_{0}\right\} .
\end{aligned}
$$

Notice that if solutions are bounded, then their derivatives are bounded as well, and therefore solutions exist for all $t \geq 0$. Moreover, if $u_{0} \leq 1, v_{0} \leq s$, and $w_{0} \leq \beta s-d$ for $d<\beta s$, then $u(t) \leq 1, v(t) \leq s$, and $w(t) \leq \beta s-d$ for all $t \geq 0$. Hence, $\mathscr{D}=\{(u, v, w): u \leq 1, v \leq s, w \leq \beta s-d\}, \beta s>d$, is positively invariant for (9).

Moreover, we obtain

$$
\dot{u} \geq u(1-u-k s)
$$

and assuming $k s<1$ we obtain $u(t)>\delta_{u}$ for any $u_{0} \geq \delta_{u}$, $\delta_{u}<1-k s$, as solutions of the logistic equation are increasing below the carrying capacity threshold. Similarly,

$$
\dot{v} \geq v\left(s-\frac{v}{\delta_{u}}-\beta^{2} s\right)
$$

and if $\beta<1$, then $v(t)>\delta_{v}$ for $v_{0} \geq \delta_{v}, \delta_{v}<s\left(1-\beta^{2}\right) \delta_{u}$. Finally,

$$
\dot{w} \geq w\left(\beta \delta_{v}-d-w\right)
$$

implying that if $d<\beta \delta_{v}$, then $w(t)>\delta_{w}$ for $w_{0} \geq \delta_{w}$ with $\delta_{w}<\beta \delta_{v}$.

Corollary 2. If $\beta s>d$, then the set $\mathscr{D}=[0,1] \times[0, s] \times[0, \beta s-$ $d]$ is positively invariant and globally attractive, while if $\beta s \leq d$, then $\mathscr{D}=[0,1] \times[0, s] \times[0, \beta s]$ is positively invariant and globally attractive for (9).

If additionally $k s<1$, then the set $\mathscr{E}=[\delta, 1] \times[0, s] \times$ $[0, \beta s-d], \beta s>d($ or $\mathscr{E}=[\delta, 1] \times[0, s] \times[0, \beta s], \beta s \leq d)$, is positively invariant and globally attractive for any $\delta<1-k s$.

If $k s<1, \beta<1$, and $d<\beta s\left(1-\beta^{2}\right)(1-k s)$, then the set $\mathscr{G}=\left[\delta_{u}, 1\right] \times\left[\delta_{v}, s\right] \times\left[\delta_{w}, \beta s-d\right]$ is positively invariant and globally attractive for any $\delta_{u}<1-k s, \delta_{v}<s\left(1-\beta^{2}\right) \delta_{u}$, and $\delta_{w}<\beta \delta_{v}-d$.

Corollary 3. If $k s<1, \beta<1$, and $d<\beta s\left(1-\beta^{2}\right)(1-k s)$, then the system described by (9) is persistent; that is, all solutions are bounded below from 0 and bounded above by some positive constants.

3.1. Local Stability of DFE and EE. Jacobi matrix for (9) reads

$J(u, v, w)$

$$
=\left(\begin{array}{ccc}
1-2 u^{2}-k v & -k u & 0 \\
\frac{v^{2}}{u^{2}} & s-2 \frac{v}{u}-\beta w & -\beta v \\
0 & \beta w & \beta v-d-2 w
\end{array}\right)
$$

For DFE we have the following relations: $1-\bar{u}-k \bar{v}=0, \bar{v}=s \bar{u}$, and hence

$$
J_{\mathrm{DFE}}=\left(\begin{array}{ccc}
-\bar{u} & -k \bar{u} & 0 \\
s^{2} & -s & -\beta \bar{v} \\
0 & 0 & \beta \bar{v}-d
\end{array}\right)
$$

implying that local stability of DFE depends on the sign of $\beta \bar{v}-d$, as submatrix $\left(\begin{array}{c}-\bar{u}-k \bar{u} \\ s^{2}-s\end{array}\right)$ generates eigenvalues with negative real parts. We easily see that $\beta \bar{v}-d>0$ is equivalent to $\beta>d k+(d / s)$, that is, to the existence of EE, according to Remark 1 .

For EE we have the relations $1-u^{*}-k v^{*}=0, s-\left(v^{*} / u^{*}\right)-$ $\beta w^{*}=0$, and $\beta v^{*}-d-w^{*}=0$, and therefore

$$
J_{\mathrm{EE}}=\left(\begin{array}{crc}
-u^{*} & -k u^{*} & 0 \\
\left(\frac{v^{*}}{u^{*}}\right)^{2} & -\frac{v^{*}}{u^{*}} & -\beta v^{*} \\
0 & \beta w^{*} & -w^{*}
\end{array}\right)
$$


Calculating characteristic polynomial for EE we obtain

$$
\begin{aligned}
P(\lambda)= & \lambda^{3}+\lambda^{2}\left(u^{*}+\frac{v^{*}}{u^{*}}+w^{*}\right) \\
& +\lambda\left(v^{*}+u^{*} w^{*}+\frac{v^{*} w^{*}}{u^{*}}+\beta^{2} v^{*} w^{*}+k \frac{\left(v^{*}\right)^{2}}{u^{*}}\right) \\
& +v^{*} w^{*}+\beta^{2} u^{*} v^{*} w^{*}+k \frac{\left(v^{*}\right)^{2} w^{*}}{u^{*}},
\end{aligned}
$$

and it is easy to see that Routh-Hurwitz criterion yields stability of EE.

Corollary 4. (I) If $\beta>d k+d / s$, then EE exists and is locally asymptotically stable.

(II) If $\beta<d k+d / s$, then EE does not exist and DFE is locally asymptotically stable.

In the original model parameters Condition (I) is $\beta r>$ $d k+r^{2} d / s$, which means that EE exists and is stable when the disease spreads with sufficiently large coefficient $\beta$, but the reproduction rate $r$ of predators cannot be large at the same time.

3.2. Global Stability. First, we find conditions for global stability of DFE in the set $\mathscr{E}$.

Theorem 5. If $\beta<k d+d / s, k<2$, and $1-k s>\delta \geq s /(2-k)$, then DFE is globally stable in $\mathscr{E}$.

Proof. We define the Lyapunov function

$$
L_{1}(u, v, w)=u-\bar{u}-\bar{u} \ln \frac{u}{\bar{u}}+v-\bar{v}-\bar{v} \ln \frac{v}{\bar{v}}+w
$$

and calculating the derivative of $L_{1}$ along the solution of (9) we get

$$
\begin{aligned}
L_{1}^{\prime}(u, v, w)= & (u-\bar{u})(1-u-k v)+(v-\bar{v}) \\
& \times\left(s-\frac{v}{u}-\beta w\right)+\beta v w-d w-w^{2} \\
\leq & -(u-\bar{u})^{2}-k(u-\bar{u})(v-\bar{v}) \\
& +(v-\bar{v}) \frac{u \bar{v}-\bar{u} v}{u \bar{u}}+(\beta \bar{v}-d) w-w^{2} .
\end{aligned}
$$

Due to the assumption on $\beta$ we have $\beta \bar{v}-d<0$, and therefore

$$
\begin{aligned}
L_{1}^{\prime}(u, v, w) \leq & -(u-\bar{u})^{2}-k(u-\bar{u})(v-\bar{v}) \\
& +(v-\bar{v}) \frac{u \bar{v}-\bar{u} v}{u \bar{u}}-w^{2} \\
\leq & -\left(\left(1-\frac{k}{2}-\frac{s}{2 u}\right)(u-\bar{u})^{2}\right. \\
& \left.+\left(\frac{1}{u}-\frac{k}{2}-\frac{s}{2 u}\right)(v-\bar{v})^{2}\right) .
\end{aligned}
$$

In $\mathscr{E}$ we have $u \in[\delta, 1]$, and hence

$$
\frac{1}{u}-\frac{k}{2}-\frac{s}{2 u} \geq 1-\frac{k}{2}-\frac{s}{2 u} \geq 1-\frac{k}{2}-\frac{s}{2 \delta} \geq 0,
$$

due to the assumptions on $k, s$, and $\delta$. This completes the proof.

Notice that for $d \rightarrow 0$ the first inequality assumed in Theorem 5 is not satisfied. This means that if the death rate of infected predators is small, then the range of stability of DFE is small as well. Moreover, as $k<2$, to have $1-k s>0$, one needs $s \leq 0.5$; for example, if $k=1$, then $s<0.5$ and $1-s>\delta \geq s$, so $\delta=0.5$ is a good choice independently of $s$.

Now, we turn to the problem of global stability of EE in $\mathscr{E}$.

Theorem 6. If $\beta>k d+d / s, k<2$, and $1-k s>\delta \geq(1-$ $\left.u^{*}\right) /\left(k(2-k) u^{*}\right)$, then EE is globally stable in $\mathscr{E}$.

Proof. We define the Lyapunov function

$$
\begin{aligned}
L_{2}(u, v, w)= & u-u^{*}-u^{*} \ln \frac{u}{u^{*}}+v-v^{*} \\
& -v^{*} \ln \frac{v}{v^{*}}+w-w^{*}-w^{*} \ln \frac{w}{w^{*}},
\end{aligned}
$$

and calculating the derivative of $L_{2}$ along the solution of (9) we get

$$
\begin{aligned}
L_{2}^{\prime}(u, v, w)= & \left(u-u^{*}\right)(1-u-k v)+\left(v-v^{*}\right) \\
& \times\left(s-\frac{v}{u}-\beta w\right)+\left(w-w^{*}\right)(\beta v-d-w) \\
= & -\left(u-u^{*}\right)^{2}-k\left(u-u^{*}\right)\left(v-v^{*}\right) \\
& +\left(v-v^{*}\right) \frac{u v^{*}-u^{*} v}{u u^{*}}-\left(w-w^{*}\right)\left(w-w^{*}\right) \\
= & -\left(u-u^{*}\right)^{2}-\frac{1}{u}\left(v-v^{*}\right)^{2}-\left(w-w^{*}\right)^{2} \\
& +\left(\frac{v^{*}}{u u^{*}}-k\right)\left(u-u^{*}\right)\left(v-v^{*}\right) \\
\leq & \left(\left(1-\frac{v^{*}}{2 u u^{*}}-\frac{k}{2}\right)\left(u-u^{*}\right)^{2}\right. \\
& +\left(\frac{1}{u}-\frac{v^{*}}{2 u u^{*}}-\frac{k}{2}\right)\left(v-v^{*}\right)^{2} \\
& \left.+\left(w-w^{*}\right)\right) .
\end{aligned}
$$

As in the proof of Theorem 5 we easily check that

$$
\frac{1}{u}-\frac{v^{*}}{2 u u^{*}}-\frac{k}{2} \geq 1-\frac{v^{*}}{2 u u^{*}}-\frac{k}{2} \geq 0
$$

due to the assumptions. Thus the proof is completed. 
Notice that as $d \rightarrow 0$ existence of the state EE is guaranteed independently of the values of $k$ and $s$. As before, the inequalities $k<2$ and $s \leq 0.5$ hold. One can check that if $d \rightarrow 0$, then the assumptions of Theorem 6 become $\beta>0$ and the others are the same as in Theorem 5 meaning that there is large set of parameter values for which EE is globally stable.

\section{Analysis of (6)}

We are looking for classical solutions of (6), and therefore we need to specify the proper space of initial conditions; cf. for example, [31]. Let $\mathbf{C}^{2+\alpha}$ denote the space of twice differentiable functions with Hölder coefficient $\alpha \in(0,1)$ and let $\mathbf{C}^{1+\alpha / 2,2+\alpha}$ denote the space $\mathbf{C}^{1+\alpha / 2}$ with respect to the time variable $t$ and $\mathbf{C}^{2+\alpha}$ with respect to the space variable $x$.

Proposition 7. Let $V(0, \cdot)=(u(0, \cdot), v(0, \cdot), w(0, \cdot)) \quad \epsilon$ $\mathrm{C}^{2+\alpha}(\bar{\Omega})$ and $u(0, \cdot)>0, v(0, \cdot) \geq 0, w(0, \cdot) \geq 0$. Then there exists unique solution of $(6)$.

Proof. Let $f(V)$ denote the vector of kinetics for (6). The function $f$ is locally Lipschitz continuous, because the coordinates $f_{i}, i=1,2,3$, are either polynomials of the second degree or rational functions well defined for $u>0$. Hence, for $V(0, \cdot) \in \mathbf{C}^{2+\alpha}$, there exists $T_{\max }>0$ such that, for every $0<T<T_{\max }$, there is a unique local solution $V \in$ $\mathrm{C}^{1+\alpha / 2,2+\alpha}(\bar{\Omega} \times[0, T])$ of (6) (compare, e.g., [31]).

Next, we would like to show nonnegativity and global existence of solutions.

4.1. Invariant Sets. In this subsection, we use the framework of invariant sets to prove global existence of solutions; compare $[32,33]$. We show that the set

$$
\mathscr{D}=[0,1] \times[0, s] \times[0, \beta s-d], \quad \beta s>d,
$$

is invariant for (6), as in the case without diffusion, that is, for (9). As before, if $\beta s \leq d$ we can substitute the last interval $[0, \beta s-d]$ with $[0, \beta s]$.

Following the ideas presented in [32] we look for the functions $G: \mathbb{R}^{3} \rightarrow \mathbb{R}$ such that $G(X) \leq 0$ in $\mathscr{D}, G$ is quasiconvex, $\nabla G$ is the left eigenvector for the diffusion coefficients matrix $D\left(D=\operatorname{diag}\left(d_{i}\right)_{i=1}^{3}\right.$ in our case $)$, and $\left.\nabla G f\right|_{\partial \mathscr{D}} \leq 0$, where $f$ denotes the right-hand side kinetic function of the studied system.

In the case of (6) studied in this section the matrix $D$ is diagonal, and hence every vector is an eigenvector for this matrix. Therefore, we can use functions $G_{i}, i=1,2,3$, which are linear as functions of appropriate variable $u, v, w$, respectively.

To show nonnegativity we use the functions

$$
\begin{aligned}
& G_{1}(V)=-u \leq 0, \\
& G_{2}(V)=-v \leq 0, \\
& G_{3}(V)=-w \leq 0, \quad \text { for } V \in \mathscr{D} .
\end{aligned}
$$

We have

$$
\begin{aligned}
& \nabla G_{1}(V) f(V)=-u(1-u-k v) \leq 0, \\
& \nabla G_{2}(V) f(V)=-v\left(s-\frac{v}{u}-\beta w\right) \leq 0, \\
& \nabla G_{3}(V) f(V)=-w(\beta v-d-w) \leq 0 \quad \text { on } \partial \mathscr{D} .
\end{aligned}
$$

Next we use

$$
\begin{aligned}
& G_{1}(V)=u-u_{M} \leq 0, \\
& G_{2}(V)=v-v_{M} \leq 0, \\
& G_{3}(V)=w-w_{M} \leq 0, \quad \text { for } V \in \mathscr{D},
\end{aligned}
$$

where $u_{M}=1, v_{M}=s$, and $w_{M}=\beta s-d, \beta s>d$ (or $w_{M}=\beta s$, $\beta s \leq d)$. For these functions we obtain

$$
\begin{aligned}
\nabla G_{1}(V) f(V) & =u(1-u-k v) \\
& \leq\left. u(1-u)\right|_{u=u_{M}}=0, \\
\nabla G_{2}(V) f(V) & =v\left(s-\frac{v}{u}-\beta w\right) \\
& \leq\left. v\left(s-\frac{v}{u_{M}}\right)\right|_{v=v_{M}}=0, \\
\nabla G_{3}(V) f(V) & =w(\beta v-d-w) \\
& \leq\left. w\left(\beta v_{M}-d-w\right)\right|_{w=w_{M}}=0 .
\end{aligned}
$$

Therefore, $\mathscr{D}$ is invariant according to the theory of invariant sets for RDEs.

The theory of invariant sets implies that, as in the case described above, if there exists a compact invariant set, then solutions of the studied system and initial data from this invariant set are global in time. This leads to the global existence of nonnegative solutions of (6) for nonnegative initial data.

In the next section, we will investigate long-time behavior of (6), including existence of global attractor and persistence property.

\section{Long-Time Behavior of Solutions of (6)}

First we focus on the persistence of the system described by (6) which is closely related to the existence of the positive equilibrium EE. We use the comparison principle for parabolic systems (cf., e.g., [34]) to show desired properties of solution of (6). As a system for comparison, we take the logistic equation with diffusion and zero-flux boundary conditions. 


\subsection{Global Attractor and Persistence Property}

Lemma 8 (see [35]). Assume that $u(x, t)$ is a solution of the problem

$$
\begin{aligned}
\frac{\partial u}{\partial t} & =d_{1} \Delta u+r u\left(1-\frac{u}{K}\right), \quad x \in \Omega, t>0, \\
\frac{\partial u}{\partial \eta} & =0, \quad x \in \partial \Omega, t>0, \\
u(x, 0) & =u_{0}(x)>0, \quad x \in \Omega ;
\end{aligned}
$$

then $\lim _{t \rightarrow \infty} u(x, t)=K$.

Lemma 9. Solutions of (6) satisfy

$$
\begin{gathered}
\limsup _{t \rightarrow \infty} \max _{x \in \bar{\Omega}} u(x, t) \leq 1, \\
\limsup _{t \rightarrow \infty} \max _{x \in \bar{\Omega}} v(x, t) \leq s, \\
\limsup _{t \rightarrow \infty} \max _{x \in \bar{\Omega}} w(x, t) \leq \beta s-d, \quad \text { for } \beta s>d, \\
\text { or } \limsup _{t \rightarrow \infty} \max _{x \in \bar{\Omega}} w(\cdot, t) \leq \beta s, \quad \text { for } \beta s \leq d .
\end{gathered}
$$

Proof. Due to nonnegativity of solutions, from the first equation of (6), we have

$$
\frac{\partial u}{\partial t} \leq d_{1} \Delta u+u(1-u)
$$

and we compare the solution of our problem with the solution of (32). Therefore, for an arbitrary $\varepsilon>0$ there exists $T \in$ $(0, \infty)$ such that $u(x, t) \leq 1+\varepsilon$ for $(x, t) \in \bar{\Omega} \times[T, \infty)$.

Next, from the second equation we have

$$
\begin{aligned}
\frac{\partial v}{\partial t} & \leq d_{2} \Delta v+v\left(s-\frac{v}{1+\varepsilon}\right) \\
& \Longrightarrow \lim _{t \rightarrow \infty} \sup _{x \in \bar{\Omega}} \max _{x \rightarrow}(x, t) \leq s(1+\varepsilon),
\end{aligned}
$$

and for any $\varepsilon_{1}>0$ there exists $T_{1} \in(T, \infty)$ such that $v(x, t) \leq$ $s\left(1+\varepsilon_{1}\right)$ in $\bar{\Omega} \times\left[T_{1}, \infty\right)$.

Finally, from the third equation, we have

$$
\frac{\partial w}{\partial t} \leq d_{3} \Delta w+w\left(\beta\left(s+s \varepsilon_{1}\right)-d-w\right)
$$

and hence

$$
\lim _{t \rightarrow \infty} \sup _{x \in \bar{\Omega}} \max _{x \in} w(x, t) \leq \beta\left(s+s \varepsilon_{1}\right)-d\left(\text { or } \leq \beta\left(s+s \varepsilon_{1}\right)\right),
$$

and the proof is completed, as $\varepsilon$ and $\varepsilon_{1}$ are arbitrary.

Theorem 10. If $k s<1, \beta s>d$, and $\beta\left(s\left(1-\beta^{2}\right)+\beta d\right)(1-k s)>$ $d$, then the system described by (6) is persistent.
Proof. Below we use upper bounds on $u, v$, and $w$ obtained in Lemma 9. From the first equation of (6), we have

$$
\begin{aligned}
\frac{\partial u}{\partial t} & \geq d_{1} \Delta u+u(1-u)-k u s(1+\varepsilon) \\
& =u(1-k s(1+\varepsilon)-u),
\end{aligned}
$$

for $\varepsilon>0$ such that $k s(1+\varepsilon)<1$. Then, by the comparison principle and Lemma 8 , we easily get $u(x, t) \geq 1-k s(1+\varepsilon)$ for $t$ large enough. As $\varepsilon$ is arbitrary, we obtain

$$
\liminf _{t \rightarrow \infty} \min _{x \in \bar{\Omega}} u(x, t) \geq 1-k s \doteq m_{1}
$$

Next, for any $\varepsilon_{2}>0$ and $t$ large enough, from the second equation, we have

$$
\frac{\partial v}{\partial t} \geq d_{2} \Delta v+v\left(s-\frac{v}{1-k s(1+\varepsilon)}-\beta\left(\beta s-d+\varepsilon_{2}\right)\right),
$$

and hence

$$
\liminf _{t \rightarrow \infty} \max _{x \in \bar{\Omega}} v(x, t) \geq(s-\beta(\beta s-d))(1-k s) \doteq m_{2} .
$$

From the third equation of (6), we have

$$
\frac{\partial w}{\partial t} \geq d_{3} \Delta w+w\left(\beta\left(m_{2}+\varepsilon_{3}\right)-d-w\right),
$$

for arbitrary small $\varepsilon_{3}>0$ and $t$ large enough. Again by the comparison principle and Lemma 8 , we have

$$
\liminf _{t \rightarrow \infty} \min _{x \in \bar{\Omega}} w(x, t) \geq \beta m_{2}-d \doteq m_{3} .
$$

Taking into account Lemma 9 we easily obtain the persistence property.

5.2. Local and Global Stability of the Positive Equilibrium EE. First, we discuss local stability of the positive equilibrium EE under the influence of diffusion.

Proposition 11. If $\beta>d k+d / s$, then the unique positive equilibrium EE of (6) exists and is locally asymptotically stable.

Proof. Linearizing (6) around EE we obtain

$$
V_{t}=\left(D \Delta+J_{\mathrm{EE}}\right) V
$$

where $V=(u(x, t), v(x, t), w(x, t))^{T}, D=\operatorname{diag}\left(d_{1}, d_{2}, d_{3}\right)$, and $J_{\mathrm{EE}}$ is the Jacobi matrix for (9) evaluated at the point $\left(u^{*}, v^{*}, w^{*}\right)$. Assuming for simplicity that $\Omega=(0, \pi)$, we have fundamental solutions of the form $e^{\lambda t} \cos \left(\mu_{i} x\right), i \in \mathbb{N}$. Therefore, for any wave number $i$ the matrix $J_{\mathrm{EE}}$ changes to

$$
J_{\mathrm{EE}}^{i}=\left(\begin{array}{ccc}
-d_{1} \mu_{i}^{2}-u^{*} & -k v^{*} & 0 \\
\frac{v^{* 2}}{u^{* 2}} & -d_{2} \mu_{i}^{2}-\frac{v^{*}}{u^{*}} & -\beta v^{*} \\
0 & \beta w^{*} & -d_{3} \mu_{i}^{2}-w^{*}
\end{array}\right),
$$


with the characteristic polynomial

$$
\begin{aligned}
P^{i}(\lambda)=\lambda^{3}+ & \lambda^{2}\left(u^{*}+d_{1} \mu_{i}^{2}+\frac{v^{*}}{u^{*}}+d_{2} \mu_{i}^{2}+w^{*}+d_{3} \mu_{i}^{2}\right) \\
+ & \lambda\left(\left(u^{*}+d_{1} \mu_{i}^{2}\right)\left(\frac{v^{*}}{u^{*}}+d_{2} \mu_{i}^{2}\right)\right. \\
& +\left(u^{*}+d_{1} \mu_{i}^{2}\right)\left(w^{*}+d_{3} \mu_{i}^{2}\right) \\
& +\left(\frac{v^{*}}{u^{*}}+d_{2} \mu_{i}^{2}\right)\left(w^{*}+d_{3} \mu_{i}^{2}\right) \\
& \left.+\beta^{2} v^{*} w^{*}+k \frac{\left(v^{*}\right)^{2}}{u^{*}}\right) \\
+ & \left(u^{*}+d_{1} \mu_{i}^{2}\right)\left(\frac{v^{*}}{u^{*}}+d_{2} \mu_{i}^{2}\right)\left(w^{*}+d_{3} \mu_{i}^{2}\right) \\
+ & \beta^{2} u^{*} v^{*} w^{*}+k \frac{\left(v^{*}\right)^{2} w^{*}}{u^{*}}
\end{aligned}
$$

and it is again easy to see that Routh-Hurwitz criterion yields stability of EE independently of the magnitude of $d_{i}, i=1,2$, 3.

In the following, we focus on global stability of EE, which implies that the three populations coexisting in the ecosystem will be spatially homogeneously distributed with increasing time.

Theorem 12. If $\beta>d k+d / s$ and $1-\left(v^{*} / 2 m_{1} u^{*}\right)-(k / 2)>0$, $m_{1}=1-k s>0$, then the unique positive equilibrium EE of (6) is globally asymptotically stable.

Proof. We construct a Lyapunov functional on the basis of the function $L_{2}$ for (9). Let us define $Z(t)=\int_{\Omega} L_{2}(u(x, t), v(x, t)$, $w(x, t)) d x$. Differentiating $Z$ along the trajectories of (6) we obtain

$$
\begin{array}{r}
\frac{d Z(t)}{d t}=\int_{\Omega}\left(\frac{\partial L_{2}}{\partial u} u_{t}+\frac{\partial L_{2}}{\partial v} v_{t}+\frac{\partial L_{2}}{\partial w} w_{t}\right) d x \\
=\int_{\Omega}\left(\frac{u-u^{*}}{u} d_{1} \Delta u+\frac{v-v^{*}}{v} d_{2} \Delta v\right. \\
\left.+\frac{w-w^{*}}{w} d_{3} \Delta w\right) d x \\
+\int_{\Omega}\left(\left(u-u^{*}\right)(1-u-k v)\right. \\
\quad+\left(v-v^{*}\right)\left(\frac{s}{b}-\frac{v}{u}-\beta w\right) \\
\left.+\left(w-w^{*}\right)(\beta v-d-w)\right) d x,
\end{array}
$$

and due to the Neumann boundary condition, we have

$$
\begin{aligned}
\frac{d E(t)}{d t}= & -\int_{\Omega}\left(d_{1} \frac{u^{*}}{u^{2}}|\nabla u|^{2}+d_{2} \frac{v^{*}}{v^{2}}|\nabla v|^{2}+d_{3} \frac{w^{*}}{w^{2}}|\nabla w|^{2}\right) d x \\
& -\int_{\Omega}\left(\left(u-u^{*}\right)^{2}+\frac{1}{u}\left(v-v^{*}\right)^{2}+\left(w-w^{*}\right)^{2}\right) d x \\
& +\int_{\Omega}\left(\left(\frac{v^{*}}{u u^{*}}-k\right)\left(u-u^{*}\right)\left(v-v^{*}\right)\right) d x \\
\leq & -\int_{\Omega}\left(d_{1} \frac{u^{*}}{u^{2}}|\nabla u|^{2}+d_{2} \frac{v^{*}}{v^{2}}|\nabla v|^{2}+d_{3} \frac{w^{*}}{w^{2}}|\nabla w|^{2}\right) d x \\
& -\int_{\Omega}\left(\left(w-w^{*}\right)^{2}+\left(1-\frac{v^{*}}{2 m_{1} u^{*}}-\frac{k}{2}\right)\left(u-u^{*}\right)^{2}\right. \\
& \left.+\left(1-\frac{v^{*}}{2 m_{1} u^{*}}-\frac{k}{2}\right)\left(v-v^{*}\right)^{2}\right) d x \leq 0
\end{aligned}
$$

which implies the desired assertion.

5.3. Local and Global Stability of Semitrivial DFE Equilibrium. Using the same approach as for the positive equilibrium we can easily show that diffusion has no influence on the dynamics of (6) also when the positive equilibrium does not exist.

Corollary 13. If $\beta<d k+d / s$ and $1-k / 2-s / 2 m_{1}>0$, $m_{1}=1-k s>0$, then the semitrivial equilibrium DFE of (6) is globally asymptotically stable.

\section{Discussion}

In the paper we have considered a prey-predator ecosystem in which the predator species is infected, such that three species (prey, healthy predators, and infected predators) are described in ecoepidemiological model based on the May predator-prey model from ecological point of view and SI model from epidemiological point of view. We have studied the influence of spatial effects incorporating simple diffusion equipped with zero-flux boundary condition into the model. Our studies included global existence and uniqueness of solutions, which is not a common procedure in the papers devoted to biological modeling but is important and can be a nontrivial, difficult issue (cf., e.g., [36]).

We have shown that the system dynamics mainly depends on the infection rate parameter $\beta$. When $\beta$ is small, there is no positive equilibrium and we expect that the population of infected predators will become extinct. This is exactly the result we obtained. We have formulated conditions for local and global stability of the semitrivial equilibrium which reflects the case of ecosystem without the disease. For $\beta$ large enough the positive equilibrium describing the disease endemic state exists and it can be expected that it is stable. In fact, there is a bifurcation at some threshold value $\beta_{\text {th }}$, such that for $\beta<\beta_{\text {th }}$ there is no positive equilibrium and the semitrivial equilibrium is locally asymptotically stable, while for $\beta$ above this threshold, the semitrivial equilibrium 
loses stability and the positive stable equilibrium exists and is stable. We have also obtained conditions on global stability of the positive equilibrium.

Our main result shows that the diffusion has no significant influence on the model dynamics; that is, local and global stability do not depend on the magnitude of diffusion coefficients. We suspect that such type of the model dynamics can be a result of simple form of diffusion incorporated into the model, as other types of diffusion can lead to more complex dynamics; cf., for example, ([34-37]). However, in our opinion, without complex studies on specific species it is difficult to recognize the law governing the process of diffusion, and therefore simple diffusion seems to be a good approximation.

\section{Conflict of Interests}

The authors declare that there is no conflict of interests regarding the publication of this paper.

\section{Acknowledgments}

This work is supported by the Fundamental Research Funds for the Central Universities, China University of Geosciences (Wuhan) (CUGL100238), and National Basic Research Program of China (973 Program) (2011CB710604,2011CB710605).

\section{References}

[1] R. M. Anderson and R. M. May, Infectious Disease of Humans Dynamics and Control, Oxford University Press, Oxford, UK, 1991.

[2] E. Venturino, "Epidemics in predator-prey models: disease in the prey," in Mathematical Population Dynamics: Analysis of Heterogeneity, O. Arino, D. Axelrod, M. Kimmel, and M. Langlais, Eds., vol. 1, pp. 381-393, 1995.

[3] S. B. Hsu and T. W. Huang, "Global stability for a class of predator-prey systems," SIAM Journal on Applied Mathematics, vol. 55, no. 3, pp. 763-783, 1995.

[4] R. M. May, Stability and Complexity in Model Ecosystems, Princeton University Press, Princeton, NJ, USA, 1973.

[5] C. S. Holling, "The functional response of predators to prey density and its role in mimicry and population regulation," Memoirs of the Entomo-Logical Society of Canada, vol. 45, supplement 45, pp. 5-60, 1965.

[6] E. Beretta, F. Solimano, and Y. Takeuchi, "Global stability and periodic orbits for two-patch predator-prey diffusion-delay models," Mathematical Biosciences, vol. 85, no. 2, pp. 153-183, 1987.

[7] E. Beretta and Y. Takeuchi, "Global stability of single-species diffusion Volterra models with continuous time delays," Bulletin of Mathematical Biology, vol. 49, no. 4, pp. 431-448, 1987.

[8] H. I. Freedman and Y. Takeuchi, "Global stability and predator dynamics in a model of prey dispersal in a patchy environment," Nonlinear Analysis. Theory, Methods \& Applications A, vol. 13, no. 8, pp. 993-1002, 1989.

[9] Y. Kuang and Y. Takeuchi, "Predator-prey dynamics in models of prey dispersal in two-patch environments," Mathematical Biosciences, vol. 120, no. 1, pp. 77-98, 1994.
[10] Z. Teng and Z. Lu, "The effect of dispersal on single-species nonautonomous dispersal models with delays," Journal of Mathematical Biology, vol. 42, no. 5, pp. 439-454, 2001.

[11] W. Wendi, C. Lansun, and L. Zhengyi, "Global stability of a population dispersal in a two-patch environment," Dynamic Systems and Applications, vol. 6, no. 2, pp. 207-215, 1997.

[12] Z. Zhang and Z. Wang, "Periodic solution for a two-species nonautonomous competition Lotka-Volterra patch system with time delay," Journal of Mathematical Analysis and Applications, vol. 265, no. 1, pp. 38-48, 2002.

[13] X. Zhang and R. Xu, "Global stability and travelling waves of a predator-prey model with diffusion and nonlocal maturation delay," Communications in Nonlinear Science and Numerical Simulation, vol. 15, no. 11, pp. 3390-3401, 2010.

[14] B. Yu, "Dynamic behavior of a plant-wrack model with spatial diffusion," Communications in Nonlinear Science and Numerical Simulation, vol. 15, no. 8, pp. 2201-2205, 2010.

[15] N. K. Vitanov, I. P. Jordanov, and Z. I. Dimitrova, "On nonlinear dynamics of interacting populations: coupled kink waves in a system of two populations," Communications in Nonlinear Science and Numerical Simulation, vol. 14, no. 5, pp. 2379-2388, 2009.

[16] E. Beretta and Y. Takeuchi, "Global asymptotic stability of Lotka-Volterra diffusion models with continuous time delay," SIAM Journal on Applied Mathematics, vol. 48, no. 3, pp. 627651,1988

[17] H. I. Freedman, B. Rai, and P. Waltman, "Mathematical models of population interactions with dispersal. II. Differential survival in a change of habitat," Journal of Mathematical Analysis and Applications, vol. 115, no. 1, pp. 140-154, 1986.

[18] Z. Y. Lu and Y. Takeuchi, "Permanence and global stability for cooperative Lotka-Volterra diffusion systems," Nonlinear Analysis. Theory, Methods \& Applications A, vol. 19, no. 10, pp. 963-975, 1992.

[19] R. Mahbuba and L. Chen, "On the nonautonomous LotkaVolterra competition system with diffusion," Journal of Differential Equations and Dynamical Systems, vol. 2, no. 3, pp. 243-253, 1994.

[20] J. Li and W. Gao, "A strongly coupled predator-prey system with modified Holling-Tanner functional response," Computers \& Mathematics with Applications, vol. 60, no. 7, pp. 1908-1916, 2010.

[21] H. I. Freedman, "Single species migration in two habitats: persistence and extinction," Mathematical Modelling, vol. 8, pp. 778-780, 1987.

[22] A. Hastings, "Dynamics of a single species in a spatially varying environment: the stabilizing role of high dispersal rates," Journal of Mathematical Biology, vol. 16, no. 1, pp. 49-55, 1982/83.

[23] I. Hanski, "Single-species metapopulation dynamics: concepts, models and observations," Biological Journal of the Linnean Society, vol. 42, no. 1-2, pp. 17-38, 1991.

[24] J. Cui, Y. Takeuchi, and Z. Lin, "Permanence and extinction for dispersal population systems," Journal of Mathematical Analysis and Applications, vol. 298, no. 1, pp. 73-93, 2004.

[25] L. Zhang and Z. Teng, "Boundedness and permanence in a class of periodic time-dependent predator-prey system with prey dispersal and predator density-independence," Chaos, Solitons and Fractals, vol. 36, no. 3, pp. 729-739, 2008.

[26] Z. Liu and S. Zhong, "Permanence and extinction analysis for a delayed periodic predator-prey system with Holling type II response function and diffusion," Applied Mathematics and Computation, vol. 216, no. 10, pp. 3002-3015, 2010. 
[27] Z. Yue and W. Wang, "Qualitative analysis of a diffusive ratiodependent Holling-Tanner predator-prey model with Smith growth," Discrete Dynamics in Nature and Society, vol. 2013, Article ID 267173, 9 pages, 2013.

[28] Y. Cai, W. Wang, and J. Wang, "Dynamics of a diffusive predator-prey model with additive Allee effect," International Journal of Biomathematics, vol. 5, no. 2, Article ID 1250023, 2012.

[29] S. Yan, X. Lian, W. Wang, and R. K. Upadhyay, "Spatiotemporal dynamics in a delayed diffusive predator model," Applied Mathematics and Computation, vol. 224, pp. 524-534, 2013.

[30] W. M. Wang, Y. L. Cai, Y. N. Zhu, and Z. G. Guo, "Alleeeffectinduced instability in a reaction-diffusion predator-prey model," Abstract and Applied Analysis, vol. 2013, Article ID 487810, 10 pages, 2013.

[31] F. Rothe, Global Solutions of Reaction-Diffusion Systems, Springer, Berlin, Germany, 1984.

[32] J. Smoller, Shock Waves and Reaction-Diffusion Equations, Springer, Berlin, Germany, 1994.

[33] K. N. Chueh, C. C. Conley, and J. A. Smoller, "Positively invariant regions for systems of nonlinear diffusion equations," Indiana University Mathematics Journal, vol. 26, no. 2, pp. 373392, 1977.

[34] Y. Giga, S. Goto, H. Ishii, and M. H. Sato, “Comparison principle and convexity preserving properties for singular degenerate parabolic equations on unbounded domains," Hokkaido University, Preprint Series in Mathematics, vol. 88, 1990.

[35] Q. Ye and Z. Li, Introduction To Reaction-Diffusion Equations, Science Press, Beijing, China, 1990.

[36] Z. B. Fang and L. Qiu, "Global existence and uniform energy decay rates for the semilinear parabolic equation with a memory term and mixed boundary condition," Abstract and Applied Analysis, vol. 2013, Article ID 532935, 12 pages, 2013.

[37] L. Zhang and S. Fu, "Nonlinear instability for a Leslie-Gower predator-prey model with cross diffusion," Abstract and Applied Analysis, vol. 2013, Article ID 854862, 13 pages, 2013. 


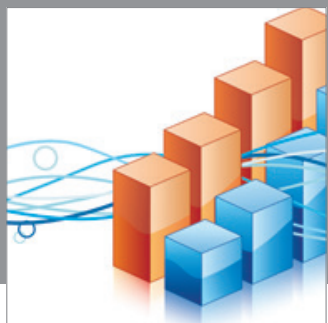

Advances in

Operations Research

mansans

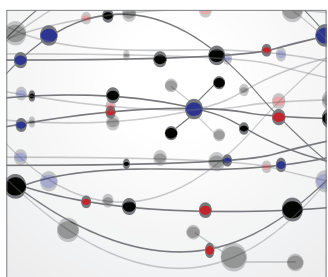

The Scientific World Journal
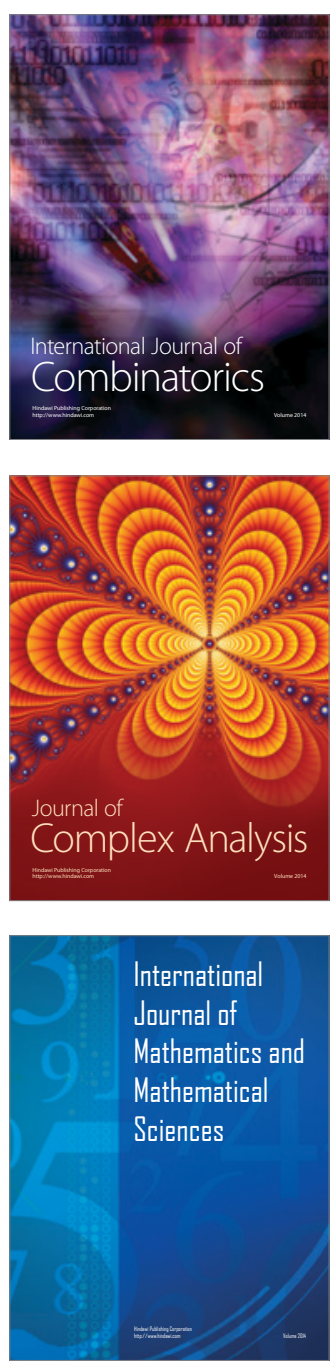
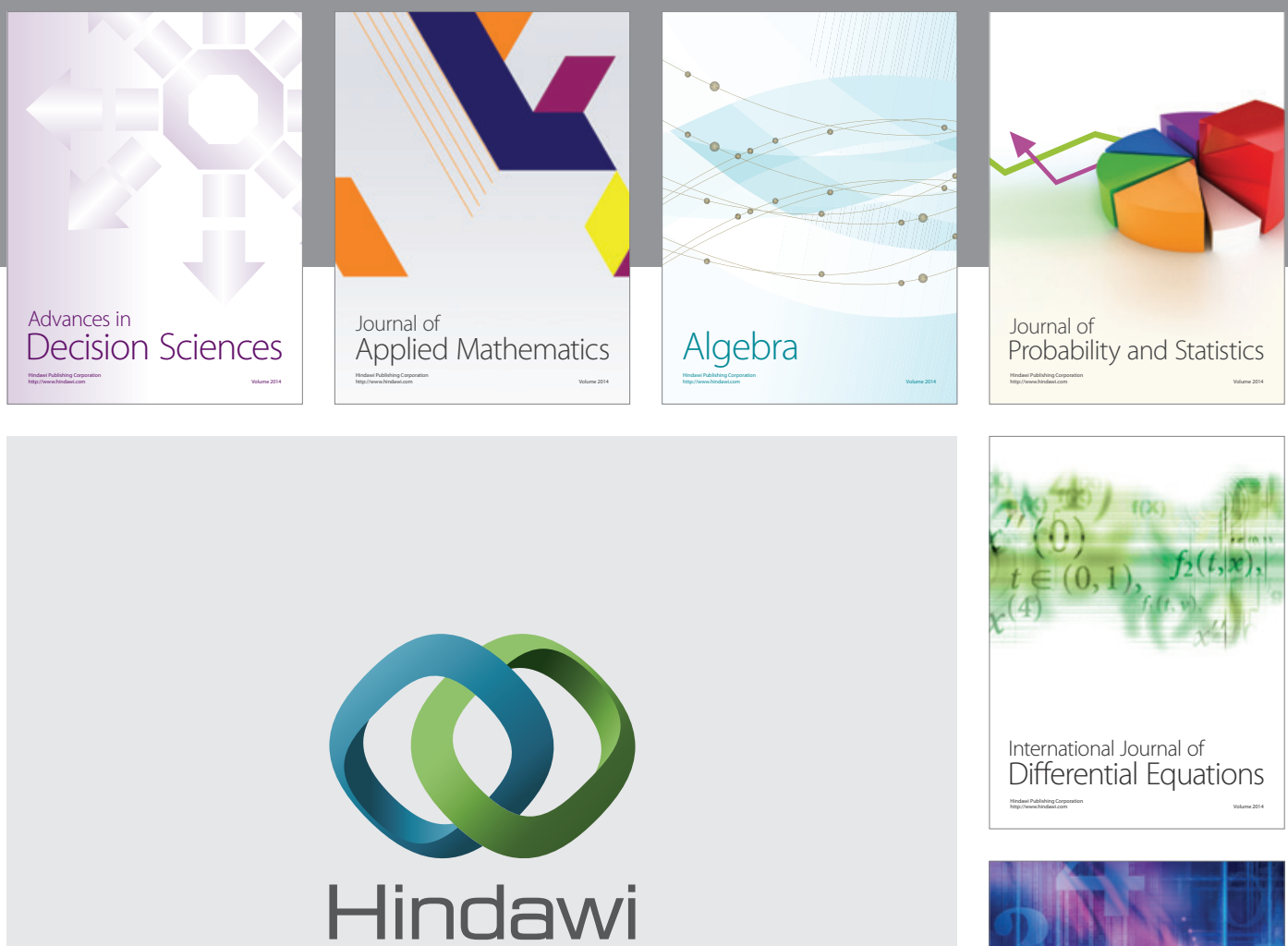

Submit your manuscripts at http://www.hindawi.com
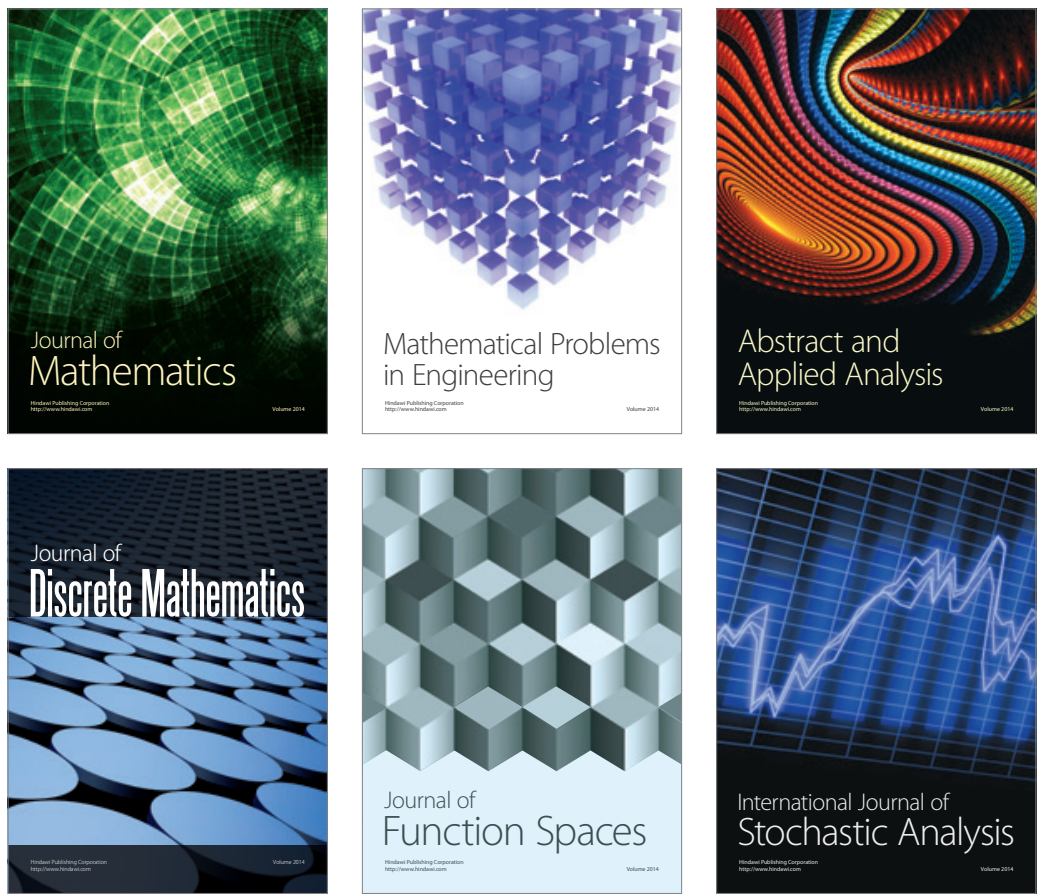

Journal of

Function Spaces

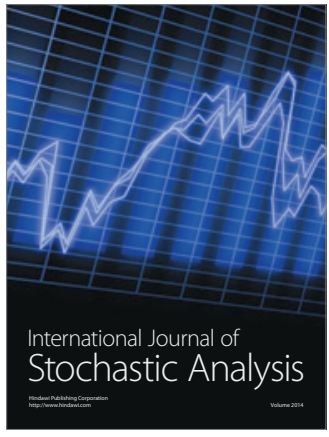

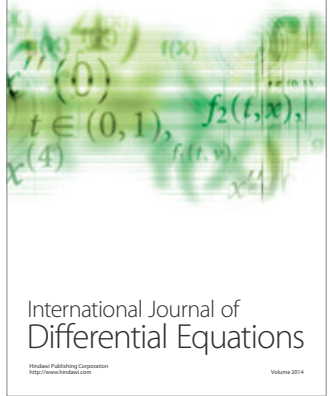
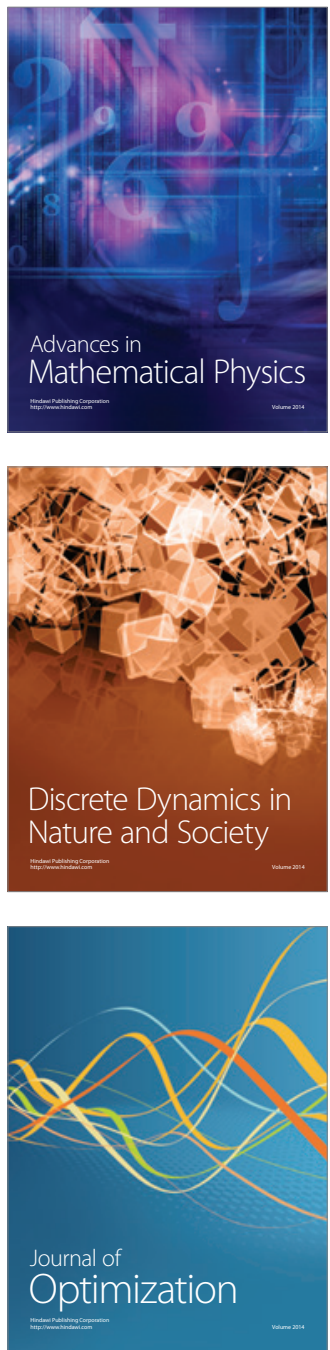